\title{
Evaluation of Oral Microflora in Precancerous Lesions
}

\author{
Dr. Esha Singh ${ }^{1}$, Dr. Ritu Garg ${ }^{2}$ \\ ${ }^{1}$ Demonstrator, Department of Oral Pathology \& Microbiology, Postgraduate Institute of Dental Sciences, Rohtak \\ ${ }^{2}$ Microbiology Dept, Govt. Medical College \& Hospital, Sec 32, Chandigarh
}

\begin{abstract}
Background: Active smoking has effects on bacterial binding to epithelial cells, expression of host cell antigens that act as receptors for some species and passive exposure to water-soluble components of cigarette smoke on bacterial binding. Passive coating of mucosal surfaces with components of cigarette smoke might enhance binding of potentially pathogenic bacteria. Aim: Our study is to evaluate the differences in the microflora of healthy individuals \& those having potentially malignant disorders. Methods: Total 80 patients were seen including 20 smokers, 20 chewers, 20 smokers as well as chewers and 20 control(non users) were neither smoker nor tobacco chewer. Three swab samples were collected of each patient i.e. one for anaerobic in Robertson cooked meat medium (enrichment medium) and streaked on BHI Agar, one for aerobic which was applied on blood agar and Mac Conkey agar and one sample for fungal culture which was applied on Sabouraud's dextrose agar. Confirmatory biochemical test were done for aerobic and anaerobic cultures and for fungal, chrome agar test were done. Results: In leukoplakia, the mean of Candida glabrata was lower whereas of other microorganisms were same. In Oral submucous fibrosis, the mean of all the microorganisms were same. In other white lesions, Candida glabrata was significantly lower in other white lesions whereas other microorganisms were same in mean count. Discussion: Our study shows no significant difference in the levels of microorganisms amongst chewers, smokers, chewers as well as smokers who did not have any clinical lesion, and even non-users of tobacco. This suggests the intricate role of these microorganisms in the development of Oral potential malignant disorders. Conclusion: Our findings also led us to suppose that the microbes are related to lesions per say and not to the etiologic factors.
\end{abstract}

Keywords: smokers, white lesions, candida, Robertson's, Sabouraud's, MacConkey

\section{Introduction}

Active smoking has effects on bacterial binding to epithelial cells, expression of host cell antigens that act as receptors for some species and passive exposure to water-soluble components of cigarette smoke on bacterial binding. Passive coating of mucosal surfaces with components of cigarette smoke might enhance binding of potentially pathogenic bacteria. Moreover, changes in the oral microflora have been well documented in oral cancer patients, and possible associations between the change of flora and the causation of cancer are being actively studied and even proved in some studies. ${ }^{1}$ It has shown that bacterial cells of oral tumours can impact signal pathways that initiate and advance oral cancer. Peptostreptococcus species have anaerobic bacteria that are commonly isolated from the smokers. Peptostreptococcus spp. and Staphylococcus aureus have been related to precancerous lesion. ${ }^{2}$ There are found in higher numbers when levels of alpha-streptococci are low due to its interfering activity against Peptostreptococcus and Staphylococcus aureus. ${ }^{1}$

Certain pathogenic strains of oral microorganisms tend to increase carcinogenic acetaldehyde concentration in saliva when metabolizing ethanol and tobacco smoke. ${ }^{3,4}$ Also, each microbiome differs in the rate at which it metabolizes the ethanol and tobacco compounds. There may be a correlation between the structure and function of the oral microbiome and oral pre-cancer and cancer. ${ }^{3}$. Hence this study was conducted to evaluate the changes in oral flora among patient with precancerous lesions.

\subsection{Aims and Objectives}

The aim of the present study was to evaluate the differences in the microflora of healthy individuals \& those having potentially malignant disorders. Since the association of pathogenic microorganisms has recently been linked to cancer, their association with precancerous lesions \& conditions might provide a vital clue to their role in the progression of neoplasia.

\section{Materials and Methods}

The study was conducted on 80 subjects visiting the Deaddiction and Rehabilitation Center of Government Medical Hospital And College,Sector-32,Chandigarh.Out of these 20 were smokers, 20 were chewers, 20 were smokers as well as chewers and 20 were control (not having any habit of chewing/smoking tobacco). All individuals included in the study were males aged between 16-45yrs of age \& were using tobacco for more than $5 y$ rs.

Samples of buccal mucosa were taken on a sterile cotton swab and were immediately transferred into the sterile test tube for the aerobic cultures. For anaerobic culture, sample were transferred immediately into Robertson cooked meat medium. The culture mediums were prepared.

The preparation of Brain Heart Infusion Agar, first agar was boiled completely and then autoclaved for 15 minutes at $121^{\circ} \mathrm{C}$. After autoclaving, the media was cooled to room temperature and sheep's blood was added approximately 10 $15 \mathrm{ml}$ in $500 \mathrm{ml}$ of medium and poured into plates. 


\section{International Journal of Science and Research (IJSR) \\ ISSN (Online): 2319-7064}

Index Copernicus Value (2016): 79.57 | Impact Factor (2015): 6.391

For Brain Heart Infusion Broth, all the ingredients were boiled till dissolved and then filtered. In Robertson cooked meat medium, prepared BHB was used for RCM. Meat particles were added to the tube and then add Ammonium nitrate BHI broth to the tubes and then tubes were autoclaved.

For Chrome Agar, the chrome agar was mixed in distilled water and boiled till dissolved. Then it was poured in petri dish and cooled it to room temperature.

New sterile swabs were used on each patient for aerobic and anaerobic cultures. The swab was rubbed for at least 10 seconds on the buccal surface, vestibule, on the dorsal surface of the tongue and the retromolar area.

For aerobic culture, the sample in the test tube was transferred on culture plate directly by the swab. Then it was kept under incubation for $12 \mathrm{hrs}$. When the growth was seen on culture plate, colour, shape and size of growth was recorded.

Catalase test was done to differentiate between Staphylococcus and Streptococcus. Using a sterile inoculating loop or wooden applicator stick, a small amount of organism was collected from a well-isolated 18- to 24hour colony and placed onto the microscope slide. Care was taken not to disturb/ pick up any agar. Using Pasteur pipette, 1 drop of $\mathrm{H}_{2} \mathrm{O}_{2}$ was placed onto the microscope slide. It was observed for immediate bubble formation $\left(\mathrm{O}_{2}+\right.$ water $=$ bubbles). \{ The reaction of peroxide with water leads to the formation of bubbles\}.The interpretation was no bubble formation (no catalase enzyme to hydrolyze the hydrogen peroxide) represented a catalase-negative reaction.

Coagulase test was done to differentiate between Staphylococcus aureus and other species of Staphylococcus. Slide Method: A clean glass slide was divided into two sections with grease pencil. One was labelled as "test" and the other as "control". A small drop of normal saline was placed on each area. With a sterilized and cooled inoculating loop, a small amount of the culture was picked up from the nutrient agar Petri plate. One or two colonies on each drop were emulsified to make a smooth suspension. The smear was about the size of a pea. With a Pasteur pipette, one drop of hydrogen peroxide was placed over the test smear. The other drop serves as control. The fluid over the smears was observed for the appearance of gas bubbles. The slide was discarded in a jar of disinfectant after the test was over.

For the anaerobic cultures, sample was immediately kept under incubation $37^{\circ} \mathrm{C}$ for $24 \mathrm{hrs}$. After 24 hours with the help of sterile inoculating loop, a small amount of sample was transferred on the anaerobic blood agar plate and then kept in anaerobic jar for $48 \mathrm{hrs}$ which ensured the anaerobic condition inside the jar by automated anaerobic system i.e. ANOXOMAT (Advanced Instruments, inc) . Colony morphology were seen on culture plate and gram staining was done. Anaerobic Gram positive organisms are likely to be Peptostreptococci.

This was followed up by the Aerotolerance test, in which aerobic culture plates as well as anaerobic culture were made. If bacteria were aerobic, growth on aerobic culture plate occurred within 24 hrs .No growth was seen if they were not aerobic. Anaerobic culture plate was checked after 48 hours. Staphylococcus aureus gives positive result. Catalase and Coagulase tests were performed to rule out any presence of microaerophilic streptococci.

Serial dilutions were prepared. Mixing the dilutions into agar plates: $100 \mathrm{ml}$ of appropriate agar was liquefied by autoclaving at $121^{\circ} \mathrm{C}$ for 15 minutes. The bottle of molten agar was placed in a $50^{\circ} \mathrm{C}$ water bath to cool down. Six empty sterile agar plates (Petri dishes) $10^{-1}$ to $10^{-6}$ were marked on the base of the plate. (Markings were avoided on lids as these could lead to confusions.)Then it was removed from the $50^{\circ} \mathrm{C}$ water bath and the outside of the bottle was wiped with tissue paper to remove water. To avoid cooling of the agar to $42^{\circ} \mathrm{C}$ (as at this temperature it sets) it was immediately poured .About $15 \mathrm{ml}$ of molten agar into each of the six plates. The agar was approximately $7 \mathrm{~mm}$ thick. 1 $\mathrm{ml}$ of each of the dilutions was pipetted into the base of correctly labelled plates using a separate pipette to avoid carryover errors. Each plate was gently swirled to mix the 1 $\mathrm{ml}$ of diluted sample into the $15 \mathrm{ml}$ of agar. The plate was left without moving for at least 15 minutes to allow the agar to set. Later the plates were incubated.

Serial dilutions from $10^{-7}$ to $10^{-6}$ were prepared \& inoculated into agar plates. After 15 minutes of incubation period, the plates for colonial growth were examined. Colonies were formed on the top of the agar as well as in the agar. Those on top of the agar were larger but all colonies were counted. The culture plates that had colonies between $25-250$ in and on the agar were selected for the best statistical representation of the number of bacteria in the undiluted sample. Plate count $x$ Dilution factor $=\mathrm{cfu} / \mathrm{ml}$. The statistical analysis was done by using SPSS software.

\section{Results}

Levels of Peptostreptococcus and Staphylococcus aureus were evaluated and the mean colony forming unit count between leukoplakia, oral submucous fibrosis and other white lesions among tobacco users and non-users were calculated.

In leukoplakia, who were non-users the mean of microorganisms like Peptostreptococcus were significantly higher. In smokers and chewers there was no significant difference. In smokers as well as chewers the mean of Peptostreptococcus were higher significantly. In total, the mean of all microorganisms were same.

In oral submucous fibrosis, who were non-users the mean of Peptostreptococcus was higher. In smokers no significant difference was seen. In chewers in descending order the mean of Staphylococcus aureus were significantly higher followed by Peptostreptococcus. In smokers as well as chewers not much difference was seen in any of the microorganisms. In total, the mean of all the microorganisms were same.

The other white lesions, in non-users, smokers and smokers as well chewers, the microorganisms count were same 


\section{International Journal of Science and Research (IJSR) ISSN (Online): 2319-7064}

Index Copernicus Value (2016): 79.57 | Impact Factor (2015): 6.391

except chewers where Staphylococcus aureus was significantly higher.

The results of our study show a significant shift of microflora in oral potentially malignant disorders. Staphylococcus aureus was found to be high in some groups, this needs to be investigated further.

Comparison of CFUs of smokers, chewers, both and nonusers of leukoplakia. Only in non-users-Candida glabrata had lesser mean value than other microorganisms and in chewers-Staphylococcus aureus had increased mean value than others microorganisms while in smokers and both(smokers as well as chewers) no significant difference could be seen. In total -the significant difference was seen only in Candida glabrata and Staphylococcus aureus in non-users and chewers respectively as shown in Table2. \& Figure 2.Comparison of CFUs of smokers, chewers, both and nonusers of leukoplakia. Only in non-users-Candida glabrata has lesser mean value than other microorganisms and in chewers-Staphylococcus aureus had increased mean value than others microorganisms while in smokers and both(smokers as well as chewers) no significant difference could be seen. In total -the significant difference was seen only in Candida glabrata and Staphylococcus aureus in non-users and chewers respectively, shown in Table3. \& Figure 3. Comparison of CFUs of smokers, chewers, both and nonusers in Other white lesions. In non user- significant difference can be seen between two species of candida ( $C$. glabrata $C$. tropicalis) and other microorganisms. While in smokers- and in both (smokers and chewers as well) Candida albicans mean value was increased and in chewersStaphylococcus aureus was increased but two species of candida (C. glabrata $C$. tropicalis) were decreased. In totalsignificant difference was seen in Candida glabrata and other microorganisms. shown in Table 4. \& Figure 4.

\section{Discussion}

The presence of an altered microflora in cancer has long been established. It has been a topic of discussion for long whether this change in microflora is associated with the pathogenesis of the disease process or is an outcome of the same. With recent studies stressing on the role of microflora in pathogenesis of cancer it has become in to further investigate. Moreover the shift in microflora in a stage wise progressive model as oral cancer might provide a vital clue to their role. The role of micro-organisms has recently gained importance, particularly with a lot of studies showing co-relation of microbes with oral squamous cell carcinoma. It is still controversial whether these microbes play a role in the in the etiology of cancer or the presence of cancer after the oral environment enough to form the growth of pathogens microbes. The relation of microbes to oral potentially malignant disorders may provide key to diagnose the precancerous lesion.

Number of Peptostreptococcus and Staphylococcus aureus has been reported to be increased in smokers. The presence of viable bacteria, mainly oral Peptostreptococcus, in the cervical lymph nodes supports the hypothesis of the association between oral mucosal damage and bacterial invasion into general circulation. It was also seen in the study that Oral submucous fibrosis cases were maximum in chewers while the incidence leukoplakia was maximum in smokers \& chewers followed by only smokers. Other white lesions were most commonly seen in non tobacco users and these findings could be due to the fact that most of these cases were clinically lichen planus.

An interesting finding was the increased incidence of lesions in individuals using tobacco for 5-10 years as compared to those using tobacco for longer periods .This was probably due to the fact that most of the subjects of our study were tobacco users for the past 5-10 years only.

Another, important finding was the increased incidence of lesions in subjects who had an increased daily frequency of tobacco usage (3 or more). This finding let us to the supposition that the frequency of tobacco usage is a more significant factor in the development of lesions than the total duration for which the patient has been smoking / chewing tobacco .This hypothesis can be justified by common sense explanation that increased frequency of tobacco usage within a short span places stress on the body with lesser chance of recoup, and therefore, more damage.

Our study showed no significant difference in the levels of microorganisms amongst chewers, smokers, chewers as well as smokers and even non-users of tobacco. This suggests that intricate role of these microorganisms in the development of Oral potential malignant disorders. These findings also let us to suppose that the microbes are related to lesions per say and not to the etiologic factors. This might be interpreted as being in favour of microorganisms colonizing the oral cavity after the development of lesion due to the changed oral environment.

The bacteria isolated from the affected site were mainly Staphylococcus aureus and Peptostreptococcus in our study. Many studies have shown that bacterial species present in blood show preferential seeding at the tumour site. Apparent diversity in the microbial taxa in both tumorous and control tissues suggests some degree of bacterial specificity.

The specificity of the bacterial species adhering to the tumour surface could be attributed to the presence of their corresponding complementary receptors with lowered immunity, resulting in the shift of oral microflora toward the unfavorable end. The other logical cause could be the irregularity of the lesion surface favoring microbial retention, especially of the anaerobes. ${ }^{5}$

Several pathogenic bacteria causing protracted course of infection can promote or initiate abnormal cell growth by demolishing the host's immune system and suppressing apoptosis. ${ }^{6}$ Intracellular pathogens survive by evading the ability of the host to identify them as non -self.

Some other species secrete toxins that can alter host cell cycles or stimulate the production of inflammatory substances causing DNA damage. ${ }^{7}$ A screening test for oral cancers based on swab collection from the site as we have done in our study or other methods like salivary count of bacterial species as shown in other researches is appealing

\section{Volume 6 Issue 12, December 2017}




\section{International Journal of Science and Research (IJSR) ISSN (Online): 2319-7064 \\ Index Copernicus Value (2016): 79.57 | Impact Factor (2015): 6.391}

${ }^{8}$ as they hold the possibility of suppression of the progress of the lesion by the use of specific antimicrobials.

Table 1: Correlation of Mean CFUs Count Of Microorganisms With Premalignant Lesion

\begin{tabular}{|c|c|c|c|c|c|c|c|}
\hline & \multirow[t]{2}{*}{$\mathrm{N}$} & \multirow[t]{2}{*}{ Mean } & \multirow{2}{*}{\begin{tabular}{|c|} 
Std. \\
Deviation \\
\end{tabular}} & \multirow[t]{2}{*}{ Std. Error } & \multicolumn{2}{|c|}{ 95\% Confidence Interval for Mean } \\
\hline & & & & & & Lower Bound & Upper Bound \\
\hline \multirow{4}{*}{$\begin{array}{l}\text { Peptostreptococcus } \\
\text { species(CFU) }\end{array}$} & Osmf & 26 & 1.2538 & .53083 & .10411 & 1.0394 & 1.4683 \\
\hline & Leukoplakia & 32 & 1.2687 & .46032 & .08137 & 1.1028 & 1.4347 \\
\hline & Other & 22 & 1.2864 & .42460 & .09052 & 1.0981 & 1.4746 \\
\hline & Total & 80 & 1.2688 & .46947 & .05249 & 1.1643 & 1.3732 \\
\hline \multirow{4}{*}{$\begin{array}{c}\text { Staphylococcus } \\
\text { aureus(CFU) }\end{array}$} & Osmf & 26 & 1.0773 & .39954 & .07836 & .9159 & 1.2387 \\
\hline & Leukoplakia & 32 & 1.2000 & .51368 & .09081 & 1.0148 & 1.3852 \\
\hline & Other & 22 & 1.2141 & .49405 & .10533 & .9950 & 1.4331 \\
\hline & Total & 80 & 1.1640 & .47185 & .05275 & 1.0590 & 1.2690 \\
\hline \multirow[t]{4}{*}{ Candida albicans (CFU) } & Osmf & 26 & 1.1846 & .34951 & .06854 & 1.0434 & 1.3258 \\
\hline & Leukoplakia & 32 & 1.3219 & .34336 & .06070 & 1.1981 & 1.4457 \\
\hline & Other & 22 & 1.2591 & .45004 & .09595 & 1.0596 & 1.4586 \\
\hline & Total & 80 & 1.2600 & .37706 & .04216 & 1.1761 & 1.3439 \\
\hline \multirow{4}{*}{$\begin{array}{l}\text { Candida tropicalis } \\
\text { (CFU) }\end{array}$} & Osmf & 26 & 1.0077 & .31231 & .06125 & .8815 & 1.1338 \\
\hline & Leukoplakia & 32 & 1.1000 & .21553 & .03810 & 1.0223 & 1.1777 \\
\hline & Other & 22 & 1.0182 & .38252 & .08155 & .8486 & 1.1878 \\
\hline & Total & 80 & 1.0475 & .29978 & .03352 & .9808 & 1.1142 \\
\hline \multirow{4}{*}{$\begin{array}{l}\text { Candida glabrata } \\
\text { ('CFU) }\end{array}$} & Osmf & 26 & .9654 & .29250 & .05736 & .8472 & 1.0835 \\
\hline & Leukoplakia & 32 & 1.0813 & .23478 & .04150 & .9966 & 1.1659 \\
\hline & Other & 22 & .9909 & .38657 & .08242 & .8195 & 1.1623 \\
\hline & Total & 80 & 1.0188 & 30193 & .03376 & .9516 & 1.0859 \\
\hline
\end{tabular}

Table 1 shows the correlation of mean CFUs count of microorganisms with premalignant lesion. Candida glabrata (CFU count) shows a significant difference between leukoplakia and OSMF \& other white lesions, but in Peptostreptococcus species, no significant difference between leukoplakia, oral submucous fibrosis and other lesion was observed. Similarly Staphylococcus aureus (CFU count), Candida albicans (CFU count) and Candida tropicalis (CFU count) no significant difference between leukoplakia, oral submucous fibrosis and other white lesion.

Table 2: Comparison of CFUs of smokers, chewers, both and non-users of leukoplakia

\begin{tabular}{|c|c|c|c|}
\hline \multicolumn{2}{|c|}{ Habit } & Mean & $\begin{array}{c}\text { Std. } \\
\text { Deviation }\end{array}$ \\
\hline \multirow{3}{*}{$\begin{array}{c}\text { Non } \\
\text { users }\end{array}$} & Peptostreptococcus species(CFU) & 1.6667 & .57735 \\
\cline { 2 - 4 } & Staphylococcus aureus (CFU) & 1.1667 & .28868 \\
\cline { 2 - 4 } & Candida albicans (CFU) & 1.1333 & .46188 \\
\cline { 2 - 4 } & Candida tropicalis(CFU) & .9333 & .46188 \\
\cline { 2 - 4 } & Candida glabrata ('CFU) & .8333 & .47258 \\
\hline Smokers & Peptostreptococcus species (CFU) & .9333 & .38816 \\
\cline { 2 - 4 } & Staphylococcus aureus(CFU) & .9333 & .33267 \\
\cline { 2 - 4 } & Candida albicans (CFU) & 1.3000 & .15492 \\
\cline { 2 - 4 } & Candida tropicalis (CFU) & 1.1000 & .08944 \\
\cline { 2 - 4 } & Candida glabrata ('CFU) & 1.0500 & .05477 \\
\hline Chewers & Peptostreptococcus species (CFU) & 1.2000 & .53385 \\
\cline { 2 - 4 } & Staphylococcus aureus (CFU) & 1.0923 & .49575 \\
\cline { 2 - 4 } & Candida albicans (CFU) & 1.1769 & .35392 \\
\cline { 2 - 4 } & Candida tropicalis (CFU) & 1.0615 & .29023 \\
\cline { 2 - 4 } & Candida glabrata ('CFU) & 1.0231 & .26818 \\
\hline Both & Peptostreptococcus species(CFU) & 1.6000 & .43205 \\
\cline { 2 - 4 } & Staphylococcus aureus(CFU) & 1.1775 & .20823 \\
\cline { 2 - 4 } & Candida albicans (CFU) & 1.0750 & .53774 \\
\cline { 2 - 4 } & Candida tropicalis (CFU) & .7500 & .44347 \\
\cline { 2 - 4 } & Candida glabrata ('CFU) & .7500 & .40415 \\
\hline Total & Peptostreptococcus species (CFU) & 1.2538 & .53083 \\
\cline { 2 - 4 } & Staphylococcus (CFU) & 1.0773 & .39954 \\
\cline { 2 - 4 } & Candida albicans (CFU) & 1.1846 & .34951 \\
\cline { 2 - 4 } & Candida tropicalis (CFU) & 1.0077 & .31231 \\
\cline { 2 - 4 } & Candida glabrata ('CFU) & .9654 & .29250 \\
\hline \multirow{7}{*}{} & & & \\
& & &
\end{tabular}

Table 2: Significant difference can be seen between two species of candida (C.glabrata C. tropicalis) and other microorganisms while in smokers-significant difference can be seen between Peptostreptococcus species \& Staphylococcus aureus and Candida species. In chewersnothing significant was seen.

Table 3: Comparison of CFUs of smokers, chewers, both and non-users of OSMF

\begin{tabular}{|c|c|c|c|}
\hline \multicolumn{2}{|c|}{ Habit } & Mean & $\begin{array}{c}\text { Std. } \\
\text { Deviation }\end{array}$ \\
\hline $\begin{array}{c}\text { Non } \\
\text { user }\end{array}$ & Peptostreptococcus species (CFU) & 1.3250 & .34034 \\
\cline { 2 - 4 } & Staphylococcus (CFU) & 1.0500 & .10000 \\
\cline { 2 - 4 } & Candida albicans (CFU) & 1.2000 & .28284 \\
\cline { 2 - 4 } & Candida tropicalis (CFU) & 1.1000 & .20000 \\
\cline { 2 - 4 } & Candida glabrata ('CFU) & .9250 & .29861 \\
\hline Smoker & Peptostreptococcus species (CFU) & 1.1778 & .57615 \\
\cline { 2 - 4 } & Staphylococcus (CFU) & 1.1222 & .56960 \\
\cline { 2 - 4 } & Candida albicans (CFU) & 1.2444 & .44472 \\
\cline { 2 - 4 } & Candida tropicalis (CFU) & 1.0333 & .22361 \\
\cline { 2 - 4 } & Candida glabrata ('CFU) & 1.0556 & .24552 \\
\hline Chewer & Peptostreptococcus species (CFU) & 1.4800 & .30332 \\
\cline { 2 - 4 } & Staphylococcus (CFU) & 1.8000 & .74833 \\
\cline { 2 - 4 } & Candida albicans (CFU) & 1.5200 & .35637 \\
\cline { 2 - 4 } & Candida tropicalis (CFU) & 1.3000 & .30822 \\
\cline { 2 - 4 } & Candida glabrata (CFU) & 1.3000 & .30822 \\
\hline Both & Peptostreptococcus species(CFU) & 1.2357 & .47004 \\
\cline { 2 - 4 } & Staphylococcus (CFU) & 1.0786 & .29917 \\
\cline { 2 - 4 } & Candida albicans (CFU) & 1.3357 & .27903 \\
\cline { 2 - 4 } & Candida tropicalis (CFU) & 1.0714 & .14899 \\
\cline { 2 - 4 } & Candida glabrata ('CFU) & 1.0643 & .13363 \\
\hline Total & Peptostreptococcus species(CFU) & 1.2687 & .46032 \\
\cline { 2 - 4 } & Staphylococcus (CFU) & 1.2000 & .51368 \\
\cline { 2 - 4 } & Candida albicans (CFU) & 1.3219 & .34336 \\
\cline { 2 - 4 } & Candida tropicalis (CFU) & 1.1000 & .21553 \\
\cline { 2 - 4 } & Candida glabrata ('CFU) & 1.0813 & .23478 \\
\hline & & & \\
\hline
\end{tabular}

Table 3: In tobacco non-users-Candida glabrata has lesser mean value than other microorganisms and in chewersStaphylococcus aureus had increased mean value than others

\section{Volume 6 Issue 12, December 2017}




\section{International Journal of Science and Research (IJSR) \\ ISSN (Online): 2319-7064 \\ Index Copernicus Value (2016): 79.57 | Impact Factor (2015): 6.391}

microorganisms while in smokers and both-(smokers as well as chewers) no significant difference could be seen.

Table 4: Comparison of CFUs of smokers, chewers, both and non-users in Other white lesions .

\begin{tabular}{|c|c|c|c|}
\hline \multicolumn{2}{|c|}{ Habit } & Mean & $\begin{array}{c}\text { Std. } \\
\text { Deviation }\end{array}$ \\
\hline Non users & Peptostreptococcus species (CFU) & 1.2769 & .44376 \\
\cline { 2 - 4 } & Staphylococcus (CFU) & 1.0692 & .25293 \\
\cline { 2 - 4 } & Candida albicans (CFU) & 1.1385 & .45376 \\
\cline { 2 - 4 } & Candida tropicalis (CFU) & .9077 & .34511 \\
\cline { 2 - 4 } & Candida glabrata ('CFU) & .9000 & .35590 \\
\hline Smokers & Peptostreptococcus species(CFU) & 1.3000 & .53852 \\
\cline { 2 - 4 } & Staphylococcus (CFU) & 1.2620 & .42157 \\
\cline { 2 - 4 } & Candida albicans (CFU) & 1.5600 & .43359 \\
\cline { 2 - 4 } & Candida tropicalis (CFU) & 1.3000 & .45826 \\
\cline { 2 - 4 } & Candida glabrata ('CFU) & 1.2000 & .50990 \\
\hline Chewers & Peptostreptococcus species (CFU) & 1.2500 & .07071 \\
\cline { 2 - 4 } & Staphylococcus (CFU) & 2.0000 & 1.41421 \\
\cline { 2 - 4 } & Candida albicans (CFU) & 1.0500 & .07071 \\
\hline
\end{tabular}
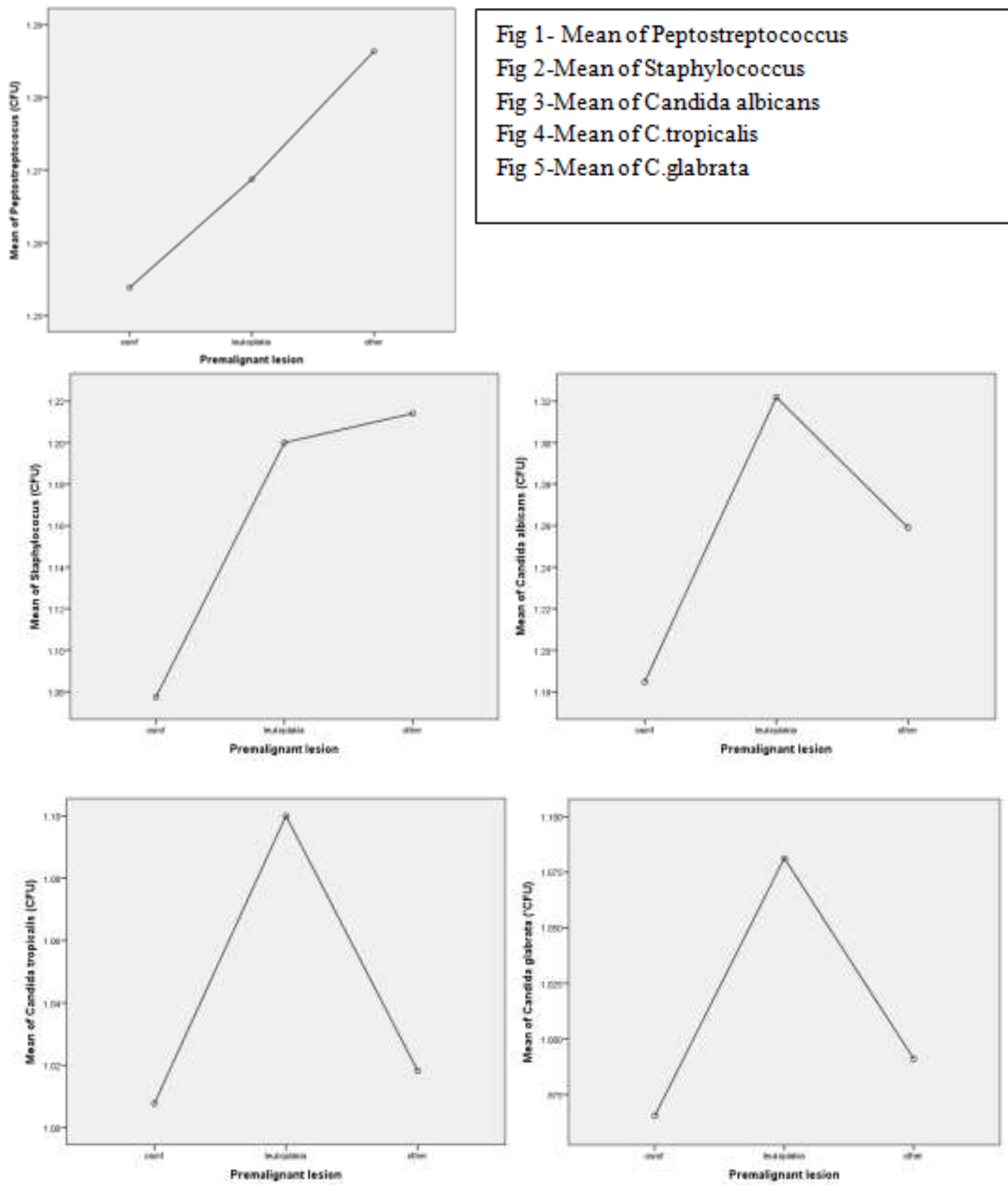

Volume 6 Issue 12, December 2017 www.ijsr.net

Licensed Under Creative Commons Attribution CC BY 


\section{International Journal of Science and Research (IJSR) \\ ISSN (Online): 2319-7064}

Index Copernicus Value (2016): 79.57 | Impact Factor (2015): 6.391

\section{References}

[1] Itzhak Brook and Alan E. Gober. -Effect of Smoking Cessation on the Microbial Flora. Arch Otolaryngology Head Neck Surg. 2007; 133:135-138.

[2] Joanne Cummins and Mark Tangney -Bacteria and tumours: causative agents or opportunistic inhabitants? Infectious Agents and Cancer 2013; 8:11.

[3] MF Zarco, TJ Vess, GS Ginsburg Oral microbiome in health and disease and the potential impact on personalized dental medicine Oral Disease.2012;18(2).

[4] Amisha A Shah, Mrinal Mayank, Aamera Farouq Mulla-Evolving role of bacteria in oral cancer. Universal Research of Journal of Dentistry.2012; 2(3) 103-106.

[5] Chocolatewala N.-The role of bacteria in Oral cancer Indian journal of Medical and Paediatric Oncology 2010;44(2):126-131.

[6] Cover-, Induction of gastric cell epithelial apoptosis by H.pylori vacuolating cytotoxin .Cancer Res 2003.

[7] Littman AJ; Chalmydia infection and risk of lung cancer .Cancer Epidemol Biomarkers Prev (13)2004.

[8] Sasaki.H -Presence of streptococcus anginosus DNA in oesophageal cancer, dysplasia of oesophagus and gastric cancer. Cancer Res 1998.

[9] Cawson RA-Leukoplakia and oral cancer, Proc R Soc Med 1969.

[10] Francesca G- Candida spp.in oral cancer and oral precancerous lesion, New microbiologica 2013; 36 283288. 